\title{
Robotic Platform Concept for Rapid Soil Monitoring
}

\section{Sergey N. BYKOV}

Ph.D. (in Engineering Sciences)

Associate Professor

Department of Agricultural Engineering

Faculty of Engineering

FSBEI of Higher Education

Kuzbass State Agricultural Academy

5, Markovtseva Str., Kemerovo, 650056, the Russian Federation

\section{Nikolay N. BEREZHNOV}

Ph.D. (in Engineering Sciences)

Associate Professor

Department of Agricultural Engineering

Faculty of Engineering

FSBEI of Higher Education

Kuzbass State Agricultural Academy

5, Markovtseva Str., Kemerovo, 650056, the Russian Federation

\author{
Alexey A. LEONOV \\ Ph.D. (in Engineering Sciences) \\ Associate Professor \\ Department of Agricultural Engineering \\ Faculty of Engineering \\ FSBEI of Higher Education \\ Kuzbass State Agricultural Academy \\ 5, Markovtseva Str., Kemerovo, 650056, the Russian Federation
}

\begin{abstract}
This article presents the substantiation of application of a robotic platform for express monitoring of the main physical-chemical and agrochemical indicators of soils in field conditions. The concept of the robotic platform provides for its movement in the field along the trajectory given by the
\end{abstract}


program, collection of soil samples and immersion of probes with sensors in them. The main advantage of the proposed method is its high efficiency at low economic costs. Due to the large number of measurements per unit area of the field the estimation accuracy is quite high. At the same time, for one point of the field it is possible to make measurements in different soil horizons.

The conceptual scheme of the robotic platform is offered, energy calculations are performed, specific brands of sensors and other devices are selected. The presented materials are the basis for designing, manufacturing and operation of this automated vehicle.

The concept of robotic platform operation corresponds to modern digital technologies and precision farming system.

Keywords: robotic platform; soil monitoring; digital technology; precision farming; sensor system

\section{Introduction}

The main directions of increasing the efficiency of agriculture are the widespread use of modern digital technologies and robotic technology (Lachuga, Izmailov, Lobachevskiy, Shogenov, 2018). One of the main methods to increase the yield and quality of agricultural products while meeting environmental requirements is recognized to be precision farming. It is a complex high-tech system of agricultural management, which includes technologies of global positioning (GPS), geographic information systems (GIS), technologies of yield assessment (Yield Monitor Technologies), technology of variable rationing (Variable Rate Technology), ERS and other technologies (Truflyak, Kramer, Kurchenko, 2017). At introduction of system of exact agriculture it is expedient to apply various robotic techniques at all stages of production process from monitoring of soils to harvesting (Brozgunova, Kochetygov, Borzykh, 2019).

\section{Methods}

In this article monographic and general scientific research methods were used. The materials for study were scientific publications in leading Russian and foreign publications. The analysis of literature sources showed that when implementing precision farming systems in agricultural production, there is a problem of collecting and analyzing data on soil characteristics for a large number of points in the field. For effective solution of this problem it is expedient to widely use modern means of automation, in particular, robotic platforms for express monitoring of soils.

\section{Literature Review}

The precision farming system provides for collecting data on the state of the vegetation cover and soil properties in elementary areas of the field (1-4 ha) for making decisions on doses of fertilizers 
and ameliorants, optimizing the timing of field work, etc. The data can be collected in different ways: satellite images, results of unmanned aerial vehicle imagery, field bypassing with different instruments. The collected data are recorded on electronic maps and then processed with the help of modern digital technologies, information systems for decision support based on a set of environmental, informational and other indicators. In hard-to-reach areas of the fields, it is necessary to use an autonomous robotic platform equipped with a system of specialized sensors and probes, as well as soil samplers for express monitoring of the main soil indicators directly in the field conditions.

We have carried out a literature review of scientific publications on possible design options and management of a robotic platform for rapid soil monitoring.

The economically effective variant for the mentioned platform is the development of its design in the form of a universal robotic means of agricultural purpose (Hort, Filippov, Kutyrev, 2019).

On this platform it is possible to install various working tools for soil monitoring as well as for traditional operations, for example, spraying plants (Bechar, Vigneault, 2017).

The modular principle of platform development allows to obtain a specialized agricultural robot (Levin, Degani, 2016).

The use of kinematic simulation methods allows to make the next step - to create a passively transformed mobile robot (Jiang, Xu, Zeng, Gao, 2019).

The principal moment when designing the platform is the choice of the mover type. We have carried out the literary review of possible types of a propeller and methods of modeling of trajectories of movement of a robotic platform corresponding to them.

The most common variant is a wheeled propeller. For example, we know the concept of all-wheel drive four-wheeled robot, which implements the extended principle of steering Ackerman (Qiu, Fan, Meng, Zhang, Zhao, 2018).

For such autonomous agricultural robots, for example, a robust digital control system is available that implements the concept of on-board turning (Fernandez, Herrera, Cerrada, 2018).

Since the robotic platform will be used in the field, it is necessary to simulate the dynamics and driving modes taking into account wheel slippage (Alipour, Robat, Tarvirdizadeh, 2019).

Analysis of slippage modes is carried out taking into account the specifics of wheel drive. There is a technique of calculation of a trajectory with variable structure of a sliding mode for the robot with a differential drive (Xue, Dong, Yan, 2018). 
In case of moving a robotic platform in particularly difficult conditions (difficult terrain, mountainous terrain, high soil humidity, etc.) it is advisable to use a tracked mover. There is a control model of the crawler robot trajectory based on the algorithm of variable sliding mode structure (Li, Chen, Zheng, Dou, Yang, 2019).

Modern mobile robot is equipped with digital motion control system. We have carried out the literary review of possible control systems of a trajectory of a robotic platform movement on a field.

There is an original online system of 3D mapping and localization for agricultural robots which can be realized in our robotic platform (Le, Omholt Gjevestad, From, 2019).

The protocol of joint routing management of the robotic platform with online adaptation can be formed according to the methodology proposed by Dusadeerungsikul and Nof (2019).

Multi-purpose planning of a robotic platform trajectory using a hybrid optimization algorithm can be performed, for example, by the PSO-MFB technology (Ajeil, Ibraheem, Sahib, Humaidi, 2020).

Hardware for autonomous navigation of the robotic platform can be made on the basis of Arduino Uno and Raspberry Pi modules (Oltean, 2019).

One of the main elements of the hardware can be a full-featured trajectory tracking controller for mobile wheeled robots (Kassaeiyan, Alipour, Tarvirdizadeh, 2020).

An important aspect of the robotic platform functioning is the motion safety provision. There is a variant of the software based on systematic cartographic logging of characteristics of investigated territories (Bozhinoski, Di Ruscio, Malavolta, Pelliccione, Crnkovic, 2019).

For orientation in space, the robotic platform must be equipped with a sensor system. We have carried out a literary review of possible spatial orientation systems for the platform.

We proposed a sensor system and environmental awareness technology for agricultural robotic vehicles (Reina, Milella, Rouveure, Nielsen, Blas, 2016).

There is an algorithm of navigation based on LiDAR for an autonomous agricultural robot that can be used on our robotic platform (Malavazi, Guyonneau, Fasquel, Lagrange, Mercier, 2018).

Ericson and Åstrand did the analysis of systems of visual odometry for use in field conditions of agriculture (Ericson, Åstrand, 2018). On the basis of this analysis it is possible to choose the most adequate system for our robotic platform.

In general, the installed sensor system can implement the method of smoothing and trajectory tracking on the basis of double DQN for robotic navigation (Zhang, Gai, Zhang, Tang, Ding, 2019). 
For express monitoring of soils it is necessary to use systems of specialized sensors and probes, as well as equipment for interpretation and recording of the received data. We have made a literature review of possible options for express soil monitoring, including those applicable to a robotic platform.

As already noted, information on soil conditions can be collected using unmanned aerial vehicles. The main method of express monitoring is photography. However, it is difficult to draw serious conclusions from such information. The variant of rounding off soil samples with the help of drones suggested in the paper is possible (Huuskonen, Oksanen, 2018). Naturally, in this case the number of samples will be insignificant.

The advantage of our robotic platform is the ability to collect relatively large amounts of samples. Rapid monitoring of the soil characteristics in the samples can be carried out, for example, with ground probes (Serrano, Ávila, Barrios, Darghan, Lobo, 2020). Subsequent evaluation of the results of express monitoring with this technology is based on the matrix potential method (Serrano et al., 2020). Decision-making about the location of sampling points in a particular field can be organized on the basis of the gradient sampling method (Liu, Zhu, Yang, Pei, Wang, 2020).

As a result of a review of scientific publications on various aspects of the design and operation of autonomous robotic vehicles for agricultural purposes to collect and study soil samples from the fields, it can be concluded that there are reasonable prerequisites for an effective design of a robotic platform for rapid soil monitoring.

\section{Results}

The main requirements for the functioning of the developed robotic platform for collection and operational study of soil samples are: increased cross-country ability, the ability to move to the specified points on the laid program, the ability to navigate in space taking into account the soil profile and field boundaries, the absence of harmful effects on the environment, the continuity of autonomous operation without recharging the batteries for at least ten hours, the possibility of collecting soil samples at a depth of $30 \mathrm{~cm}$, the possibility of express evaluation.

Taking into account the literature review and the abovementioned basic requirements we have developed the concept of a robotic platform for express soil monitoring. The concept diagram of the robotic platform is shown in Figure 1. 

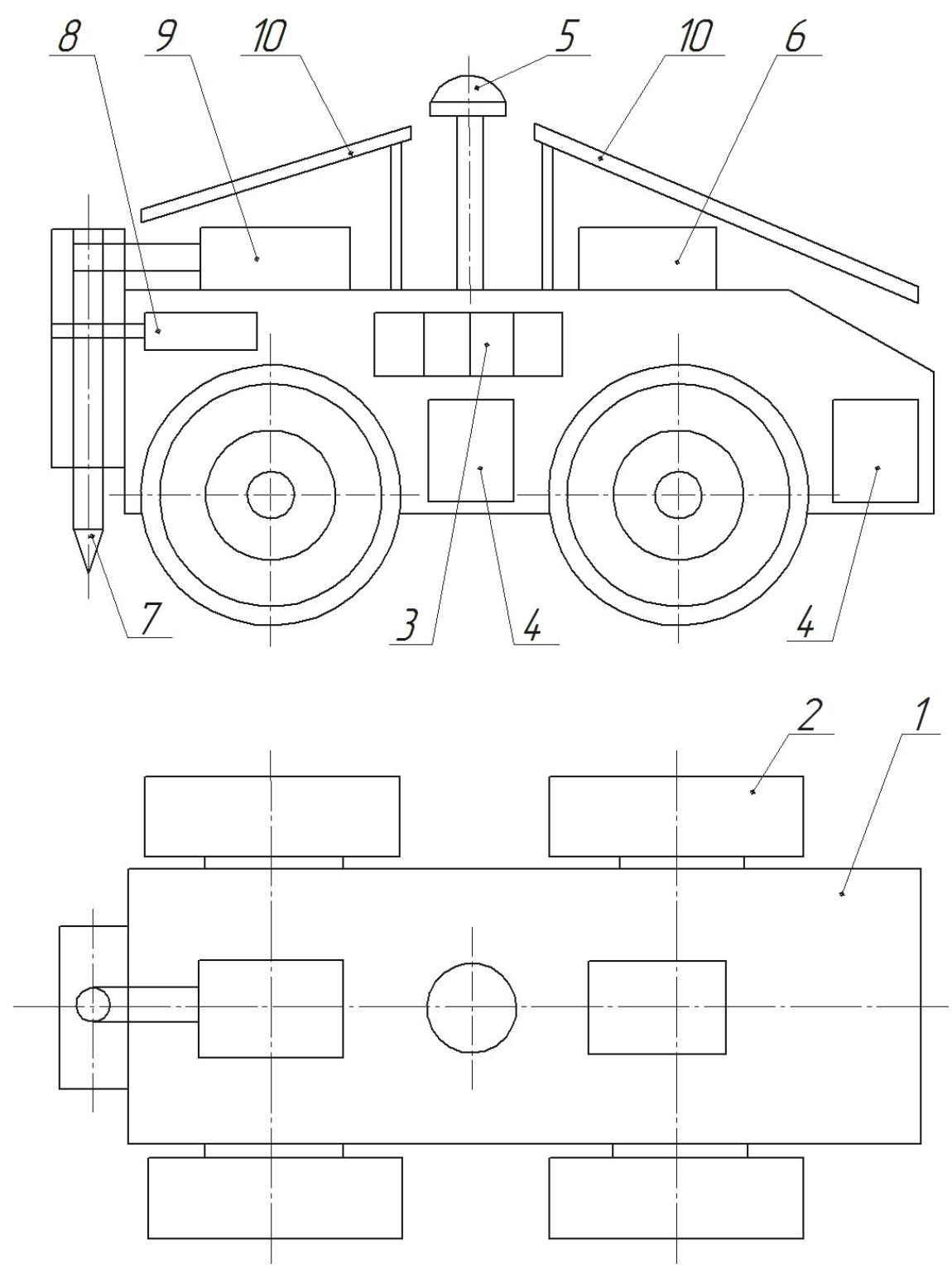

Figure 1. Scheme of a robotic platform for rapid soil monitoring

\section{1 - frame; 2 - wheel; 3 - wheel engine; 4 - battery pack; 5 - transport sensor unit; 6 - central} control unit of the platform and the working body; 7 - working body; 8 - engine of the working body; 9 - sensor unit; 10 - solar batteries

The robotic platform has a support frame 1 on which all actuators, diagnostic equipment and control equipment are installed.

The field is driven by a $4 \times 4$ wheel drive. Each wheel 2 has an independent drive, which provides high cross-country ability and maneuverability of the machine. Wheel rotation is provided by DC motors 3 (not shown on the top view). Electric motors 3 are powered by the battery block 4 (not shown on the top view).

Orientation of the platform in space is carried out using a block of transport sensors 5, which includes several video cameras, radar, lidars, GPS-module and others. In fact, this unit performs the 
function of receiving and transmitting information about the platform location, driving speed, presence of obstacles on the field surface and on the horizon.

Transport sensor unit 5 communicates with the central control unit 6 . The latter, in addition to the platform motion control function, controls the working device 7 installed at the front of the platform and designed for soil sampling. Rotating and deepening of the drill of the working device is provided by a DC motor 8 , which is powered by the battery block 4 .

The soil sample taken is delivered to the upper part of the working device 8 , where the probes of the express sensors are immersed in it. The information from the sensors is processed and stored in the sensor control unit 9.

Additional power supply to the platform is provided by installing solar panels 10 (not shown on top).

For the proposed robotic platform concept, the following tasks had to be solved in the first phase:

- In view of the wheeled mover already selected, a review of the existing structures of the agricultural wheeled robots should be carried out in order to identify their design features, strengths and weaknesses, which will be taken into account when designing our platform;

- to determine in advance the main structural dimensions of the robotic platform;

- to perform preliminary calculations of resistance forces to movement of the robotic platform on different agrophones and engine power;

- review existing soil sampling units and develop our own soil sampling unit design, taking into account the above requirements for our robotic platform;

- select specific brands of sensors and other instruments for rapid soil characterization monitoring to be installed on our robotic platform.

- Consider installing them on our solar platform.

Most of the work on the above list has already been done by us.

A review of existing designs of agricultural wheeled robots has been carried out. We analyzed the designs of wheeled agricultural robots: Adigo Field Flux Robot (Norway); BoniRob developed by Amazon (Germany); RM View developed at Carnegie Malon University (USA); FarmWise developed by designers from San Francisco (USA); AGRowBot developed by Emerging Technology (USA). The individual technical solutions implemented in these robot engines are of interest when designing our robotic platform.

The following preliminary dimensions of the platform under development have been determined: 
- dimensions (length $2.2 \mathrm{~m}$, width $1.0 \mathrm{~m}$, height $1.3 \mathrm{~m}$ );

- wheelbase $1.1 \mathrm{~m}$;

- track width $0.7 \mathrm{~m}$;

- wheel diameter $0.6 \mathrm{~m}$;

- $\quad$ wheel width $0.2 \mathrm{~m}$;

- ground clearance $0.2 \mathrm{~m}$

Preliminary energy calculations have been made. A significant influence on the performance of our robotic platform is the resistance force caused by the interaction of its running systems with the field surface. This is most relevant when the platform is moving through various soil agrophones, which are characterized by a wide range of physical and mechanical properties.

Rolling resistance of a robotic platform is composed of private resistance to movement of all its propellers. For the $4 \times 4$ wheel arrangement that we have adopted, rolling resistance is calculated only for the leading propulsors.

At definition of forces of resistance to rolling of wheels of a robotic platform we accept a case of uniform movement of a wheel with the elastic tyre on a deformable surface as such is soil. We accept also that the surface is horizontal, friction force in a wheel hub and resistance of air environment are absent. In this case, the energy supplied to the wheel is spent on three types of work that make up the total energy of rolling resistance of the wheel:

- vertical pressing of the soil and the formation of a compacted track (track);

- the elastic deformation of the tyre causing internal friction in the tyre material;

- tire friction against the road surface in the contact spot.

Pneumatic wheels of our robotic platform for improved traction on the ground are equipped with soil trailers. In this case, the following forces act when the drive wheel interacts with the ground:

- the friction force between the tyre support surface and the ground;

- the shear force that occurs when the tyre hitches are resting on the ground;

- the force of the ground cut by the side edges of the load hooks.

On paved roads, friction forces are essential. On loose soils, the last two components increase significantly and in many cases are decisive. In this case, the wheel bearing surface adheres to the ground mainly due to the forces of shearing and shearing of the ground layers clamped between the ground hooks. 
The tangential force of the wheel traction is equal to the sum of the tangential reactions of the soil on each individual grouser when the ground is deformed in the direction opposite to that of the robotic platform.

$$
\mathrm{P}_{\mathrm{K}}=\sum_{1}^{n_{\mathrm{T}}} \mathrm{P}_{\mathrm{k.cд}}+\sum_{1}^{n_{\mathrm{T}}} \mathrm{P}_{\mathrm{k.} \mathrm{ср}},
$$

where $\mathrm{P}_{\mathrm{k} \text {. сд }}$ - shear force, $\mathrm{kH} ; \mathrm{P}_{\mathrm{k} . \mathrm{cp}}$ - shearing force, $\mathrm{kH} ; n_{\mathrm{r}}$ - number of ground grousers, that are in contact with the soil within the contact length.

We get an expression that defines tangential force of wheel traction as the sum of two components the force required to deform the ground shear and the force that occurs when the soil layers are cut by the side edges of the tire grouser, $\mathrm{kH}$.

$$
\mathrm{P}_{\mathrm{r}}=\frac{f_{\mathrm{ck}} k_{\tau} G_{\mathrm{r}}}{\delta L}\left[\ln \operatorname{ch} \frac{\delta L}{k_{\tau}}-f_{\mathrm{mp}}\left(\frac{1}{\operatorname{ch} \frac{\delta L}{k_{\tau}}}-1\right)\right]+2 \tau_{\mathrm{cp}} \frac{h_{\mathrm{r}} L}{t},
$$

where $f_{\mathrm{ck}}, f_{\text {пр }}-$ sliding friction coefficient and the given quiescent friction coefficient respectively; $k_{\tau}, \tau_{\mathrm{cp}}$ - deformation coefficient $(\mathrm{m})$ and shear stress (module) respectively $(\mathrm{H} / \mathrm{m}) ; G_{\mathrm{K}}$ - normal tire load , $\mathrm{kH} ; \delta$ - propeller slipping, $\% ; L$ - wheel length, $\mathrm{m} ; t, h_{\mathrm{r}}$ - the distance between the star wheels and their height respectively, $\mathrm{m}$.

Thus, the tangential force of elastic wheel traction required to overcome the forces of friction, shear and shear of the soil depends on the normal load $G_{\mathrm{K}}$, acting on the wheel, the parameters of the wheel (profile width $B$, diameter $D$, istance and height of the ground $t$ nd $h_{\mathrm{r}}$ ), physical and mechanical properties of the soil (coefficients of sliding and rest friction $f_{\mathrm{ck}}$ and $f_{\mathrm{m}}$, deformation and volume buckling of the soil $k_{\tau}$ and $k$, the limit of compressive strength $\sigma_{0}$ ) the mode of wheel movement (coefficient of slipping $\delta$ ).

At a substantiation of parameters of the robotic platform propulsor we calculate for medium loamy soils on three most typical for agricultural production agrophones - virgin soil, grain stubble and cultivated soil, which will provide the greatest coverage of the range on the conditions of operation of the structure. 
IV International Scientific and Practical Conference "Modern S\&T Equipments and Problems in Agriculture"

Value of slipping of thrusters we accept $14 \%$ on the basis of established agrotechnical requirements to agricultural machinery with $4 \times 4$ wheel scheme.

The results of calculation of components of tangential force of wheel traction are shown in Figure 2.

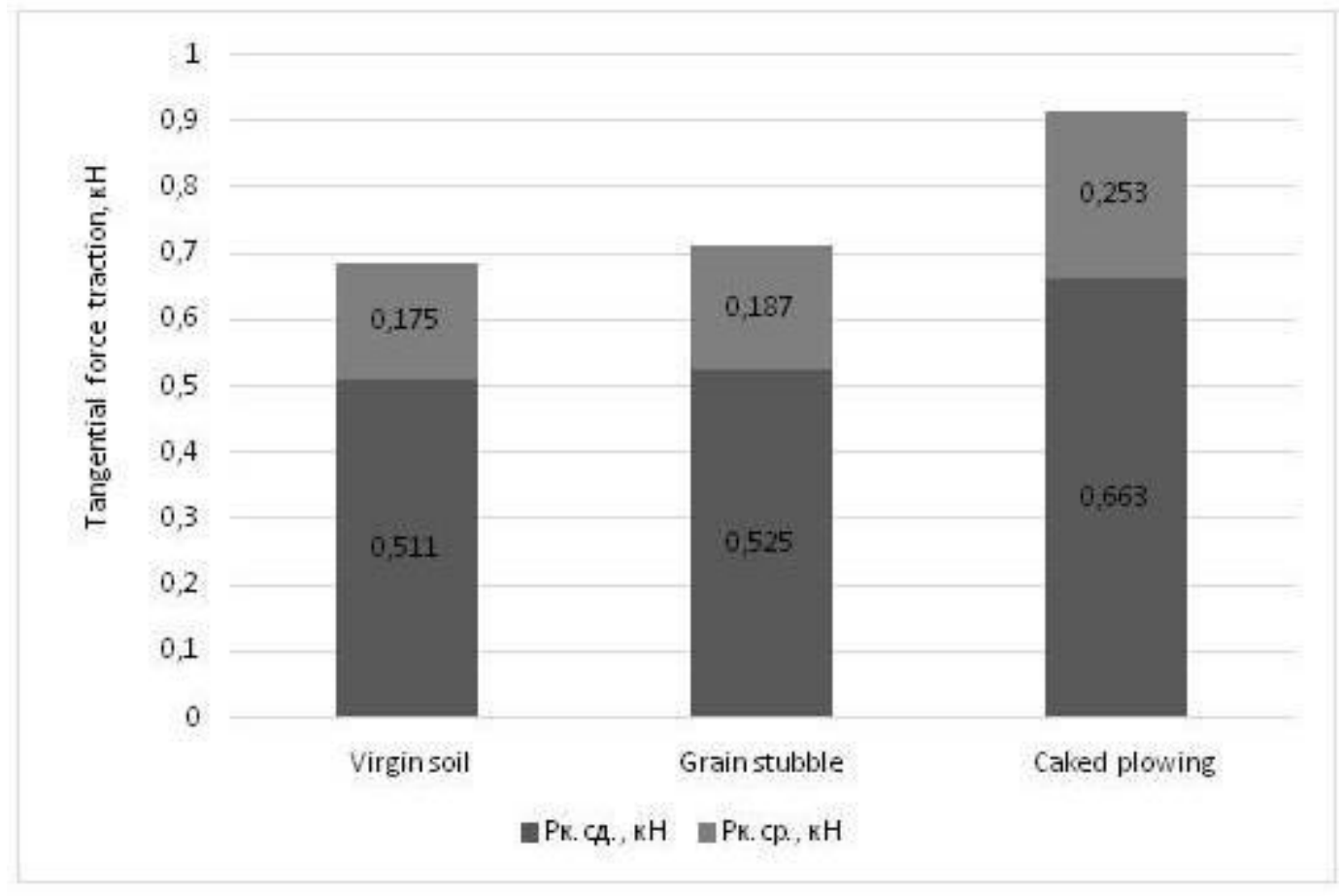

Figure 2. Components of tangential force traction wheels

Based on the traction balance equation, it is possible to record the total rolling resistance of the wheels of a robotic platform:

$$
P_{f}=n_{\mathrm{K}} \mathrm{P}_{\mathrm{K}} \text {, }
$$

where $n_{\mathrm{K}}$ - number of driving wheels.

The results of calculations for the three main agrophones of the robotic platform movement are shown in Figure 3. 


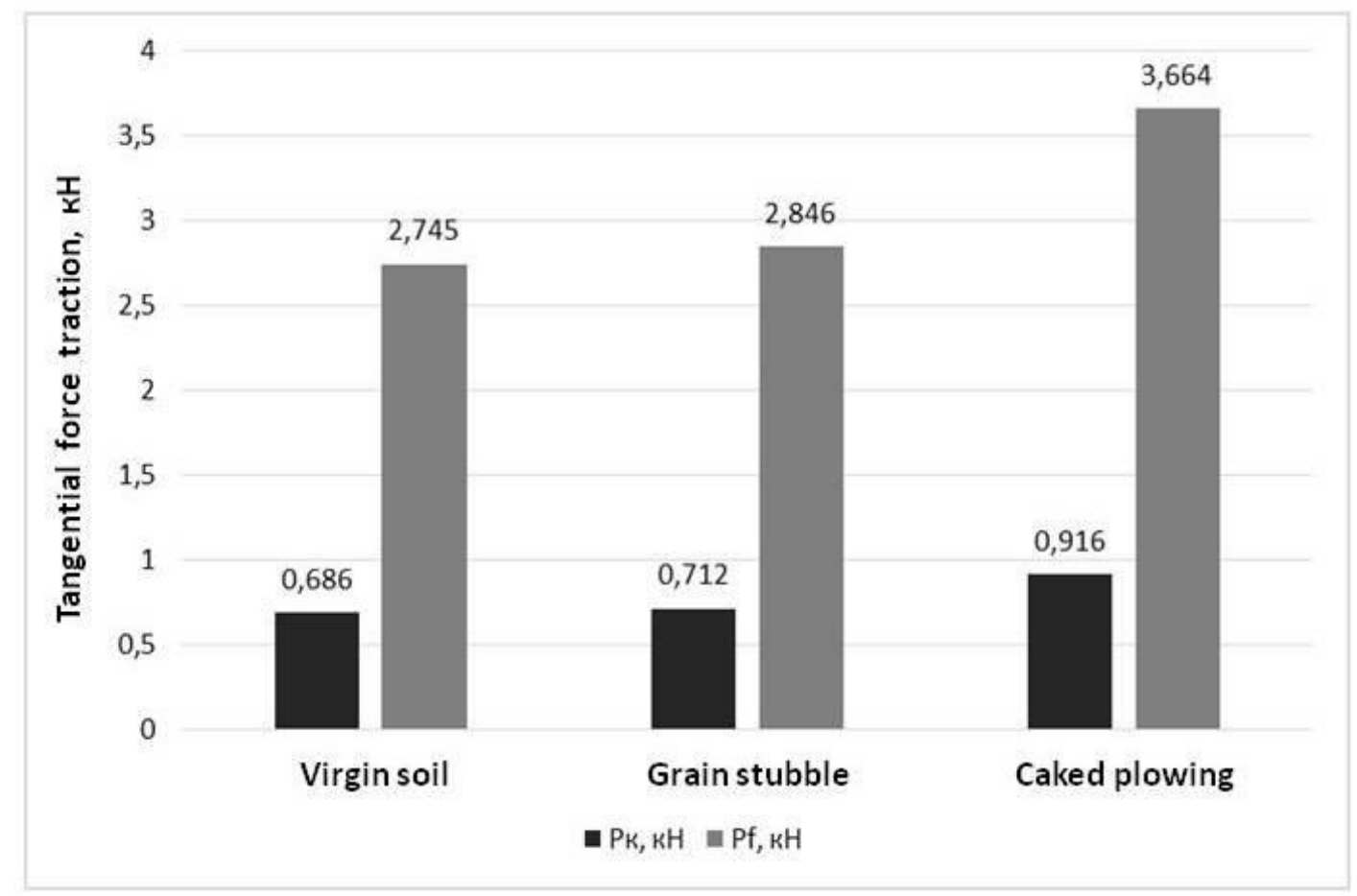

Figure 3. The strength of resistance to moving a robotic platform for different soil preparation

On the basis of resistance force calculations made for different movement conditions of a robotic platform, it is possible to predict and substantiate the energy characteristics of its power unit - rated effective power and motor type.

We have calculated the required engine power of a robotic platform with a maximum weight of about $700 \mathrm{~kg}$, moving in difficult field conditions (existing ploughing).

We calculate the total maximum capacity of the robotic platform motors using the following formula

$$
N_{\max }=(F * G) \frac{V_{\max }}{\eta_{m p} * \eta_{3 \partial} * \eta_{x \partial}}
$$

where $F$ - wheel resistance coefficient; $G$ - total weight of the robotic platform with the equipment, $\mathrm{kH} ; V_{\max }$ - maximum driving speed, $\mathrm{m} / \mathrm{s} ; \eta_{m p}-$ transmission efficiency; $\eta_{\ni \partial}$ - net promoter efficiency; $\eta_{\kappa \partial}-$ efficiency of the installed wheeled propulsor.

$$
N_{\max }=(0,035 * 6,88) \frac{3}{0,9 * 0,9 * 0,8}=1,12 \mathrm{kB \textrm {T }}
$$

Since each wheel has an individual on-board engine, it is necessary to calculate the power of each wheel by the formula 


$$
N_{\partial \epsilon}=\frac{N_{\max }}{K_{\partial \epsilon} * \eta_{\tilde{\sigma} p}}
$$

where $K_{\partial \varepsilon}-$ total onboard engine capacity ; $\eta_{\ni \partial}-$ the efficiency of the drive when installing multiple motors.

$$
N_{\partial \epsilon}=\frac{1,12}{4 * 0,5}=0,56 \mathrm{kBT}
$$

According to the results of calculations, we install on our robotic platform four DC motors type MPK-104, having a nominal speed of $1500 \mathrm{rpm}$ and power of $0.6 \mathrm{~kW}$.

\section{Discussion}

We have analyzed the design of soil samplers: Amity Concord A2450 (USA); Nietfeld Duoprob 60 (Germany); Multiprob 120 (Germany); Wintex 1000 (Denmark) and others. Separate technical solutions implemented in the considered soil samplers were of interest in the design of our design. The design of the soil collector developed by us is already being manufactured, and a set of documents for patent application has been prepared.

Specific brands of probes, sensors and other instruments for rapid monitoring of soil characteristics were selected. The following sensors are to be installed on the robotic platform under development for rapid monitoring of soil physical and chemical properties:

- sensor ETR-111 with a probe for measuring soil acidity;

- sensor MS-7828 with two probes for soil moisture measurement;

- ADA Thermotester 330 sensor with a probe for soil temperature measurement;

- complex probes ZD-06 and PH-300 with probes for soil acidity and humidity measurement;

- TR-46908 complex transducer with two probes for soil moisture and temperature measurement;

For express monitoring of nitrogen, potassium and phosphorus content in soil it is planned to install the following devices:

- Luster Leaf Rapitest 1880 device for agrochemical indicators measurement;

- device Fertometr for measuring agrochemical indicators;

- multi-monitor Rapitest mini 18 for soil fertility measurement;

- ionomer I-510 for measuring ion concentration. 
All these instruments will be adapted to measure soil characteristics directly in the field, and the soil data collected will be recorded on an electronic medium georeferenced.

One of the important aspects of a robotic platform is its power supply. One environmentally friendly way to solve the problem is to use solar panels. We have reviewed agricultural robots that use solar energy.

The following robots were considered: Ladybird, developed at the University of Sydney (Australia); RIPPA, developed by the Australian Center for Field Robotics; Zoe, developed by NASA and Carnegie Mellon University (USA); GROVER, developed by NASA; Kubota X Tractor by Kubota (Japan). Technical decisions on installation of solar batteries, realized in the specified robots, are of certain interest. On the developed robotic platform it is possible to install a solar panel of about 1 sq.m. Such a battery will have a capacity of about $300 \mathrm{~W}$. This will amount to $10-15 \%$ of the total power consumption of the robot's engines, mechanisms and devices.

\section{Conclusion}

Analysis of physico-chemical and agro-chemical indicators of soil is an obligatory condition for obtaining high yields and preserving the fertility of used lands. Traditional methods of evaluation of soil indicators, consisting in the selection of soil samples with subsequent laboratory research, are characterized by high time and economic costs.

For the analysis of most indicators is possible option of express monitoring, which consists of immersion in a soil sample of probes with sensors. The whole set of probes and sensors is installed on a robotic platform moving along the field along the trajectory defined by the program. This method is characterized by high speed and low economic costs. Due to the large number of measurements per unit of field area (ten times more than with conventional methods) the accuracy of evaluation is significantly improved. Besides, for one point of the field it is possible to make measurements in different soil horizons.

The proposed concept of a robotic platform for rapid monitoring of soils in the field, the developed concept of the platform, energy calculations, the developed design of the soil sampler, selected specific brands of sensors and other devices are the basis for the design, manufacture and operation of this automated vehicle.

The use of the proposed robotic platform will increase the efficiency of agricultural production. 


\section{References}

Ajeil, F.H., Ibraheem, I.K., Sahib, M.A., Humaidi, A.J. (2020). Multi-objective path planning of an autonomous mobile robot using hybrid PSO-MFB optimization algorithm. Applied Soft Computing, $89, \quad 106076 . \quad$ Retrieved from: https://www.sciencedirect.com/science/article/abs/pii/S1568494620300168?via\%3Dihub

Alipour, K., Robat, A.B., Tarvirdizadeh, B. (2019). Dynamics modeling and sliding mode control of tractor-trailer wheeled mobile robots subject to wheels slip. Mechanism and Machine Theory, 138, 16-37. Retrieved from: https://www.sciencedirect.com/science/article/abs/pii/S0094114X18319062?via\%3Dihub

Bechar, A., Vigneault, C. (2017). Agricultural robots for field operations. Part 2: Operations and systems. Biosystems Engineering, 153, 110-128. Retrieved from: https://www.sciencedirect.com/science/article/abs/pii/S1537511015301926?via\%3Dihub

Bozhinoski, D., Di Ruscio, D., Malavolta, I., Pelliccione, P., Crnkovic, I. (2019). Safety for mobile robotic systems: A systematic mapping study from a software engineering perspective. Journal of Systems and Software, 151, 150-179. Retrieved from: https://www.sciencedirect.com/science/article/pii/S0164121219300317?via\%3Dihub

Brozgunova, N.P., Kochetygov, A.I., Borzykh, A.A. (2019). Prospects of robotics usage in agroindustrial complex. Science and Education, 2, 312.

Dusadeerungsikul, P.O., Nof, S.Y. (2019). A collaborative control protocol for agricultural robot routing with online adaptation. Computers \& Industrial Engineering, 135, 456-466. Retrieved from: https://www.sciencedirect.com/science/article/abs/pii/S0360835219303675?via\%3Dihub

Ericson, S.K., Åstrand, B.S. (2018). Analysis of two visual odometry systems for use in an agricultural field environment. Biosystems Engineering, 166, 116-125. Retrieved from: https://www.sciencedirect.com/science/article/abs/pii/S1537511016305189?via\%3Dihub

Fernandez, B., Herrera, P.J., Cerrada, J.A. (2018). Robust digital control for autonomous skidsteered agricultural robots. Computers and Electronics in Agriculture, 153, 94-101. Retrieved from: https://www.sciencedirect.com/science/article/pii/S016816991830783X?via\%3Dihub 
IV International Scientific and Practical Conference "Modern S\&T Equipments and Problems in Agriculture"

Hort, D.O., Filippov, R.A., Kutyrev, A.I. (2019). Development of the universal robotic means for an agricultural purpose (in Russian). Journal of Advanced Research in Technical Science, $13,69-73$.

Huuskonen, J., Oksanen, T. (2018). Soil sampling with drones and augmented reality in precision agriculture. Computers and Electronics in Agriculture, 154, 25-35. Retrieved from: https://www.sciencedirect.com/science/article/pii/S0168169918301650?via\%3Dihub

Jiang, H., Xu, G., Zeng, W., Gao F. (2019). Design and kinematic modeling of a passively-actively transformable mobile robot. Mechanism and Machine Theory, 142, 103591. Retrieved from: https://www.sciencedirect.com/science/article/abs/pii/S0094114X19300266?via\%3Dihub

Kassaeiyan, P., Alipour, K., Tarvirdizadeh, B. (2020). A full-state trajectory tracking controller for tractor-trailer wheeled mobile robots. Mechanism and Machine Theory, 150, 103872. Retrieved from: https://www.sciencedirect.com/science/article/abs/pii/S0094114X20300938?via\%3Dihub

Lachuga, Yu.F., Izmailov, A.Yu., Lobachevskiy, Ya.P., Shogenov, Yu.H. (2018). Intensive machine technologies, the robotized technique and the digital systems for manufacture of the basic groups of agricultural production. Machinery and equipment for rural areas, 7, 2-7.

Le, T., Omholt Gjevestad, J.G., From, P.J. (2019). Online 3D Mapping and Localization System for Agricultural Robots. IFAC-Papers OnLine, 52(30), 167-172. Retrieved from: https://www.sciencedirect.com/science/article/pii/S2405896319324358?via\%3Dihub

Levin, M., Degani, A. (2016). Design of a Task-Based Modular Re-Configurable Agricultural Robot. IFAC-Papers OnLine, 49(16), 184-189. Retrieved from: https://www.sciencedirect.com/science/article/pii/S240589631631597X?via\%3Dihub

Li, Z., Chen, L., Zheng, Q., Dou, X., Yang, L. (2019). Control of a path following caterpillar robot based on a sliding mode variable structure algorithm. Biosystems Engineering, 186, 293306.

Retrieved

from: https://www.sciencedirect.com/science/article/abs/pii/S1537511019307810?via\%3Dihub

Liu, X., Zhu, A., Yang, L., Pei, T., Wang, D. (2020). A graded proportion method of training sample selection for updating conventional soil maps. Geoderma, 3571, 113939. Retrieved from: https://www.sciencedirect.com/science/article/pii/S0016706117305256?via\%3Dihub

Malavazi, F.B., Guyonneau, R., Fasquel, J.B., Lagrange, S., Mercier, F. (2018). LiDAR-only based navigation algorithm for an autonomous agricultural robot. Computers and Electronics in 
IV International Scientific and Practical Conference "Modern S\&T Equipments and Problems in Agriculture"

Agriculture, 154, 71-79. $\quad$ Retrieved from:

https://www.sciencedirect.com/science/article/pii/S0168169918302679?via\%3Dihub

Oltean, S.E. (2019). Mobile Robot Platform with Arduino Uno and Raspberry Pi for Autonomous Navigation. Procedia Manufacturing, 32, 572-577. Retrieved from: https://www.sciencedirect.com/science/article/pii/S2351978919302896?via\%3Dihub

Qiu, Q., Fan, Z., Meng, Z., Zhang, Q., Zhao, C. (2018). Extended Ackerman Steering Principle for the coordinated movement control of a four wheel drive agricultural mobile robot. Computers and Electronics in Agriculture, 152, 40-50. Retrieved from: https://www.sciencedirect.com/science/article/pii/S0168169917315922?via\%3Dihub

Reina, G., Milella, A., Rouveure, R., Nielsen, M., Blas, M.R. (2016). Ambient awareness for agricultural robotic vehicles. Biosystems Engineering, 146, 114-132. Retrieved from: https://www.sciencedirect.com/science/article/abs/pii/S1537511015001889?via\%3Dihub

Serrano, D., Ávila, E., Barrios, M., Darghan, A., Lobo, D. (2020). Surface soil moisture monitoring with near-ground sensors: Performance assessment of a matric potential-based method. Measurement, 155, $107542 . \quad$ Retrieved from: https://www.sciencedirect.com/science/article/pii/S0263224120300798?via\%3Dihub

Truflyak, E.V., Kramer, A.S., Kurchenko, N.Yu. (2017). Precision agriculture: yesterday, today, tomorrow. British Journal of Innovation in Science and Technology, 2(4), 15-26.

Xue, J., Dong, S., Yan, J. (2018). Sliding Mode Variable Structure Based Path Following Control of Agricultural Robots with Differential Drive. IFAC-Papers OnLine, 51(17), 455-459. Retrieved from: https://www.sciencedirect.com/science/article/pii/S2405896318312916?via\%3Dihub

Zhang, W., Gai, J., Zhang, Z., Tang, L., Ding, Y. (2019). Double-DQN based path smoothing and tracking control method for robotic vehicle navigation. Computers and Electronics in Agriculture, 166, $104985 . \quad$ Retrieved from: https://www.sciencedirect.com/science/article/pii/S0168169919302066?via\%3Dihub 